\title{
DECOMPOSITION THEORIES FOR ABELIAN CATEGORIES
}

\author{
BY
}

\author{
JOE W. FISHER AND HARVEY WOLFF
}

\begin{abstract}
Both the classical approach to decomposition theories and Fisher's technique of constructing decomposition theories from radical functions are extended to and exploited in the context of abelian categories. These two different approaches to decomposition theories for abelian categories intertwine in one theorem from which flows necessary and sufficient conditions for the existence of the tertiary, primary, and Bourbaki's 9 -primary decomposition theories.
\end{abstract}

Introduction. In [3] a new technique was developed for constructing decomposition theories for modules. This technique was used in [3] to yield necessary and sufficient conditions for the existence of the Lesieur-Croisot tertiary decomposition theory and in [4] to yield necessary and sufficient conditions for the existence of the classical Lasker-Noether primary decomposition theory. We show that this technique can be extended in order to produce decomposition theories for certain abelian categories.

Our approach to decomposition theories in $\delta_{1}$ is via associated element functions with values in a partially ordered set. We define radical functions and associated element functions on admissible subcategories of abelian categories. From a radical function $r$ on an admissible subcategory $\mathcal{C}$, we construct an associated element function $A$ on $C$. We define $A$-subobjects and $A$-decompositions. Theorem 1.3 then yields necessary and sufficient conditions for this associated element function $A$, which is obtained from $r$, to be a decomposition theory on $\mathcal{C}$. In $\$ 1$ we give several examples of radical functions.

Our approach to decomposition theories in $\$ 2$ is the classical approach via radical functions. In order to do this we introduce a way in which concepts like primary submodules, primary decompositions, tertiary submodules, and tertiary decompositions can be handled in the context of abelian categories. This is achieved by using a pair of radical functions $r_{1}$ and $r_{2}$ to define $r_{1}-r_{2}$ subobjects and $r_{1}-r_{2}$ decompositions. These two different approaches to decomposition theories intertwine in Theorem 2.1. As corollaries we get necessary and sufficient conditions for the existence of the Lesieur-Croisot tertiary decomposi-

Presented to the Society, September 5, 1972; received by the editors January 7, 1972. AMS (MOS) subject classifications (1970). Primary 13A15, 16A64, 16A66, 18 E99.

Key words and phrases. Decomposition theories, abelian categories, radical functions. 
tion theory (Corollary 2.2), for the classical Lasker-Noether primary theory (Corollary 2.3), and for Bourbaki's $\mathcal{P}$-primary decomposition theory (Corollary 2.6). Also we obtain Corollary 2.9 which asserts that an admissible subcategory $\mathcal{C}$ of $R$-modules where $R$ is a commutative Noetherian ring with unity has Bourbaki's $\mathcal{P}$-primary theory if and only if Ass $(M)$ is finite for each $M$ in $\mathcal{C}$.

1. In this section we review some basic definitions, results and examples of decomposition theories in the context of abelian categories. Since the proofs of the results mentioned are the same or are slight modifications of the proofs in [3], we refer the reader to that paper for more details.

Throughout we assume that $B$ is an $A B-5$ abelian category which is well powered, although many of the results can be shown to hold with less restrictions on the category. A full, nonempty subcategory $\mathcal{C}$ of $B$ is called admissible (a) if $M \in \mathcal{C}$ and $0 \rightarrow M^{\prime} \rightarrow M \rightarrow M^{\prime \prime} \rightarrow 0$ exact in $\mathbb{B}$ then $M^{\prime}$ and $M^{\prime \prime} \in \mathcal{C}_{\text {; }}$ (b) if $M_{1}$, $M_{2} \in \mathcal{C}$ then $M_{1} \oplus M_{2} \in \mathcal{C}$. So any Serre subcategory of $\mathfrak{B}$ is admissible.

Let $X$ be a partially ordered set and $\mathcal{C}$ an admissible subcategory of $B$. Then a radical function on $\mathcal{C}$ with values in $X$ is a function which assigns to each $M \in \mathcal{C}$ an element $r(M) \in X$ such that if $0 \rightarrow M^{\prime} \rightarrow M$ is exact with $M \in \mathcal{C}$ then $r(M) \leq r\left(M^{\prime}\right)$. As an example, let $B$ be $A B-5$ with a generator $P$. Let $C=B$ and $X$ be the set of subobjects of $P$. Define $r(M)=\bigcap\{\operatorname{Ker} f \mid f: P \rightarrow M\}$. It is clear that $r$ is a radical function.

An object $M \neq 0$ of $\mathcal{C}$ is called $r$-stable if for every exact sequence $0 \rightarrow$ $M^{\prime} \rightarrow M, r\left(M^{\prime}\right)=r(M)$. If $M \in \mathcal{C}$, then an element $x \in X$ is an associated element of $M$, if there exists an $r$-stable subobject $S$ of $M$ such that $x=r(S)$. We let $A(M)$ be the set of associated elements of $M$. We call $A$ the associated element function on $\mathcal{C}$ that is obtained from $r$. Then the following results are shown in $[3,2.6$, p. 245$]$.

Proposition 1.1. Let $M \in \mathcal{C}$. If $x \in A(M)$ then there exists a subobject $N(x)$ of $M$ such that

(1) $A(M / N(x))=\{x\}$,

(2) $A(N(x))=A(M)-\{x\}$.

Theorem 1.2. If every nonzero subobject of $M$ in $C$ contains an r-stable subobject then there exists a decomposition of 0 in $M$ of the form $\varphi=\bigcap_{x \in A(M)} N(x)$ where $A(M / N(x))=\{x\}$ for $x \in A(M)$.

Now $M \in \mathcal{C}$ is called $A$-stable if $M \neq 0$ and there exists an $x \in X$ with $A(M)=$ $\{x\}$. Note that if $M$ is $r$-stable then $M$ is $A$-stable. If $0 \rightarrow N \rightarrow M$ is exact then $N$ is an $A$-subobject of $M$ if $M / N$ is $A$-stable. We say $M \in C$ is $A$-finite if $A(M)$ is a finite set. A finite set $\left\{N_{i}: i \in I\right\}$ of subobjects of $M \in C$ is an A-decomposition of $N$ in $M$ if 
(1) $\bigcap_{i \in I} N_{i}=N$ and for no $i \in I$ is $\bigcap_{j \neq i} N_{j} \subseteq N_{i}$;

(2) each $N_{i}$ is an $A$-subobject of $M$; and

(3) $A\left(M / N_{i}\right) \neq A\left(M / N_{j}\right)$ for $i \neq j$.

An object $M \in \mathcal{C}$ is said to be worthy of a decomposition theory with respect to $r$ or just $r$-worthy if each quotient $M^{\prime \prime}$ of $M$ satisfies

(a) each nonzero subobject of $M^{\prime \prime}$ contains an $r$-stable subobject, and

(b) $A\left(M^{\prime \prime}\right)$ is finite.

Let $\mathcal{C}$ be an admissible subcategory for the radical function $r$. Then $A$ is a decomposition theory on $\mathcal{C}$ if for each $M \in \mathcal{C}, 0$ has an $A$-decomposition in $M$.

Theorem 1.3. [3, Theorem 4.10, p. 252]. Let $r$ be a radical function on an admissible category $\mathcal{C}$ and let $A$ be the associated element function on $\mathcal{C}$ that is obtained from $r$. Then $A$ is a decomposition theory on $\mathcal{C}$ if and only if each object in $\mathcal{C}$ is r-worthly.

We end this section with some examples.

Example 1. Let $R$ be a ring, let $R$ M denote the category of left $R$-modules and $R$-homomorphisms, and let $\mathcal{C}$ be an admissible subcategory of ${ }_{R} M$. For $M \epsilon$ $C$ let $a(M)$ be the annibilator of $M$, i.e., $a(M)=(0: M)=\{x \in R: x M=0\}$. Then $a$ is a radical function on $\mathcal{C}$.

Example 2. Let $R, R$, and $\mathcal{C}$ be as in Example 1. For $M \in \mathcal{C}$ let $t(M)$ be the tertiary radical of $M$, i.e., $t(M)=\{x \in R$ : there exists an essential submodule $E$ of $M$ with $x E=0$ \}. Then $t$ is a radical function on $\mathcal{C}$ by [3, Lemma 2.1]. Let $T$ be the associated element function on $\mathcal{C}$ which is obtained from $t[3, \delta 5]$.

Theorem 1.3 shows that $T$ is a decomposition theory on $\mathcal{C}$ if and only if each object in $\mathcal{C}$ is $t$-worthy.

Example 3. Let $R, R^{M}$, and $\mathcal{C}$ be as in Example 1. For $M \in \mathcal{C}$ let $p(M)$ be the primary radical of $M$, i.e., the intersection of all the prime ideals in $R$ which contain $(0: M)$. Then $p$ is a radical function on $\mathcal{C}$. Let $P$ be the associated element function on $\mathcal{C}$ which is obtained from $p[4, \$ 1]$. Theorem 1.3 shows that $P$ is a decomposition theory on $\mathcal{C}$ if and only if each object in $\mathcal{C}$ is p-worthy.

Example 4. Let $R$ be a commutative ring with unity and let $\mathcal{C}$ be an admissible subcategory of ${ }_{R} M$. For $M \in \mathcal{C}$ let $w(M)$ be the weakly primary radical of $M$, i.e., $w(M)=\left\{x \in R\right.$ : for each $m \in M$ there exists a positive integer $n$ with $x^{n} m=$ $0\}[3, \S 10]$. Then $w$ is a radical function on $\mathcal{C}$. Let $W$ be the associated element function on $\mathcal{C}$ which is obtained from $w$. Theorem 1.3 shows that $W$ is a decomposition theory on $\mathcal{C}$ if and only if each object in $\mathcal{C}$ is $w$-worthy.

Example 5. Let $\mathfrak{B}$ be a locally Noetherian abelian category. Then by [2] every injective object is a direct sum of indecomposable injectives. Let $X$ be a partially ordered set with infs. Then we can define a radical function $r$ on $B$ by first defining $r(U)$ where $U$ is an indecomposable injective. Then if $E(A)$ is the 
injective envelope of $A, E(A)=\Sigma_{i \in I} \bigoplus U_{i}$ where $U_{i}$ is an indecomposable injec tive. Hence we can set $r(A)=\bigwedge_{i \in l} r\left(U_{i}\right)$. That this is a radical function follows easily from the fact that if $0 \rightarrow M_{1} \rightarrow M$ is exact in $B$ then $E\left(M_{1}\right) \subseteq E(M)$ and the $U_{i}$ appearing in the decomposition for $E\left(M_{1}\right)$ appear among the $U_{i}$ appearing in the decomposition for $E(M)[2, \mathrm{p} .163]$. If $\mathcal{C}$ is the full subcategory of $\mathcal{B}$ whose objects are the Noetherian objects of $\mathcal{B}$, then $\mathcal{C}$ is admissible and it can be easily shown that every object in $\mathcal{C}$ is $r$-worthy. Hence by Theorem $1.3, \mathcal{C}$ has the $A$-decomposition theory.

Example 6. Let $Y$ be a Hausdorff space, $R$ a sheaf of rings on $Y$ and $M$ the category of sheaves of $R$-modules. Then $M$ is an AB-5 abelian category with a generator, and so is locally small [6]. For the set $X$ we take the set of subsheaves of $R$. We show that given a radical function on $\mathbb{N}$ we get one on the category of $R_{y}$-modules $\left(R_{y}\right.$ is the stalk of $R$ at $\left.y\right)$. Conversely, given radical function $r_{y}$ on $R_{y}$-modules for all $y \in X$, these generate a radical function on $\mathbb{N}$.

Lemma. (a) Let $\mathcal{C}$ be an admissible subcategory of $\mathbb{M}$. Then for every $y \in X$, $\mathcal{C}_{y}=\left\{C_{y} \mid C \in \mathcal{C}\right\}$ is an admissible subcategory of $\pi_{y} \cdot\left(\mathbb{M}_{y}\right.$ is the category of $R_{y^{-}}$ modules.)

(b) If for all $y, \mathfrak{T}_{y}$ is an admissible subcategory of $\mathbb{M}_{y}$ then $\left\{\mathscr{D}_{y}\right\}=\{C \in \mathbb{M} \mid$ $C_{y} \in \mathfrak{T}_{y}$ for all $\left.y \in X\right\}$ is admissible in $\mathbb{M}$.

(c) $\mathcal{C} \subseteq\left\{\mathcal{C}_{y}\right\} ; \mathcal{C}_{y}=\left\{\mathcal{C}_{y}\right\}_{y}$.

Proof. (a) It is clear that $\mathcal{C}_{y}$ is closed under direct sums. Suppose that (1) $0 \rightarrow A \rightarrow C_{y} \rightarrow B \rightarrow 0$ is exact. Considering $\{y\} \subseteq Y$ as a subspace we have that $(1)$ is an exact sequence of sheaves. Denoting by $i D$ the extension of $D$ by zero we get the following two exact sequences of sheaves on $\{y\}$.

$$
\begin{aligned}
& 0 \rightarrow C_{Y-\{y\}} \rightarrow C \rightarrow i C_{y} \rightarrow 0, \\
& 0 \rightarrow i A \rightarrow i C_{y} \rightarrow i B \rightarrow 0 .
\end{aligned}
$$

From (2) we conclude that $i C_{y} \in \mathcal{C}$ and from (3) that $i A$ and $i B \in \mathcal{C}$. So $A=i A_{y} \epsilon$ $\mathcal{C}_{y}$ and $B=i B \in \mathcal{C}_{y}$.

(b) and (c) are clear.

Proposition. (a) Let $\mathcal{C}$ be an admissible subcategory of $M$ and $r$ a radical function with values in $X$, the subsheaves of $R$. Then $r_{y}$, defined on $\mathcal{C}_{y}$ by $r_{y}\left(C_{y}\right)=r\left(i C_{y}\right)$, is a radical function on $C_{y}$.

(b) If for all $y \in Y, r_{y}$ is a radical function on $\mathfrak{D}_{y}$ with values in the submodules of $R_{y}$, then there exists a radical function $\left\{r_{y}\right\}$ on $\left\{D_{y}\right\}$ with values in $X$.

(c) If $r$ is a radical function on $\mathcal{C}$ in $\pi$ such that if $0 \rightarrow A \rightarrow B \rightarrow C \rightarrow 0$ is exact in $\mathcal{C}$ then $r(B) \leq r(C)$, then for every $C \in \mathcal{C}{ }_{r}(C) \leq\left\{r_{y}\right\}(C)$. 
Proof. (a) If $0 \rightarrow A \rightarrow B$ is exact in $C_{y}$ then $0 \rightarrow i A \rightarrow i B$ is exact in $C$. Hence $r(i B) \leq r(i A)$ so $r_{y}(B)=r(i B)_{y} \leq r(i A)_{y}=r_{y}(A)$.

(b) If $H_{y} \in \prod_{y}$ for all $y$, let $P\left(\left\{H_{y}\right\}\right)$ be the sheaf whose value at the open set $U$ is $\mathrm{II}_{x \in U} H_{x}$ (see [5]). Now let $D \in\left\{\mathfrak{I}_{y}\right\}$. Then for every $y \in Y, 0 \rightarrow r_{y}\left(D_{y}\right) \rightarrow$ $R_{y}$ is exact. So there exists an exact sequence $0 \rightarrow P\left(\left\{r_{y}\left(D_{y}\right)\right\}\right) \rightarrow P\left(\left\{R_{y}\right\}\right.$. Now there exists a canonical monomorphism of sheaves $R \stackrel{a}{\rightarrow} P\left(\left\{R_{y}\right\}\right)$. We define $\left\{r_{y}\right\}(D)$ to be the following pullback:

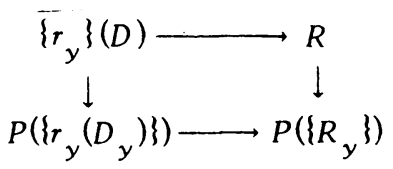

It is clear that $\left\{r_{y}\right\}$ is a radical function on $\left\{\mathscr{T}_{y}\right\}$.

(c) Now $r(C) \leq r\left(i C_{y}\right)$ for all $y$. Hence the following diagram commutes for all $y$.

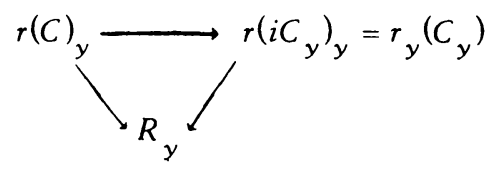

So we get the following commutative diagram.

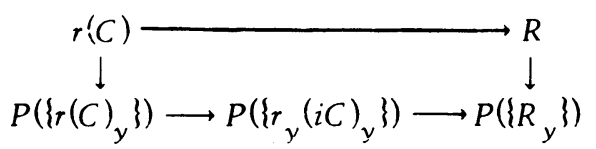

Consequently there exists a map of subsheaves of $R$ from $r(C) \rightarrow\left\{r_{y}\right\}(C)$. Thus $r(C) \leq\left\{r_{y}\right\}(C)$.

2. In this section we introduce a way in which concepts like primary submodules, primary decompositions, tertiary submodules, and tertiary decompositions can be handled in the context of abelian categories.

Let $\mathcal{B}$ be as in $\oint_{1}$ and let $\mathcal{C}$ be an admissible subcategory of $\mathcal{B}$. Let $r_{1}$ and $r_{2}$ be radical functions on $\mathcal{C}$ with values in $X$ such that $r_{1} \leq r_{2}$, i.e., for each $C \in \mathcal{C}, r_{1}(C) \leq r_{2}(C)$. A subobject $N$ of an object $M \in \mathcal{C}$ is called an $r_{1}-r_{2}$ subobject if $N \neq M$ and if for each nonzero subobject $M^{\prime \prime}$ of $M / N, r_{1}\left(M^{\prime \prime}\right) \leq r_{2}(M / N)$. A finite set $\left\{N_{i}: i \in I\right\}$ of subobjects of $M \in \mathcal{C}$ is an $r_{1}-r_{2}$ decomposition of a subobject $N$ of $M$ if the following are satisfied:

(1) $\bigcap_{i \in I} N_{i}=N$ and for no $i \in I$ is $\bigcap_{j \in i} N_{j} \subseteq N_{i}$;

(2) the $N_{i}, i \in I$, are $r_{1}-r_{2}$ subobjects of $M$; and

(3) $r_{2}\left(M / N_{i}\right) \neq r_{2}\left(M / N_{j}\right)$ for $i \neq j$.

We say that $\mathcal{C}$ has the $r_{1}-r_{2}$ decomposition theory if for each $M \in \mathcal{C}, 0$ has an $r_{1}-r_{2}$ decomposition in $M$.

Example 7. Let $R, R_{R}^{M}$, and $\mathcal{C}$ be as in Example 1. First we note that $a \leq$ $t$ on $\mathcal{C}$. If $M \in \mathcal{C}$, then $N$ is an $a-t$ submodule of $M$ if and only if $N$ is a ter- 
tiary submodule of $M\left[3\right.$, p. 243]. Moreover, a finite set $\left\{N_{i}: i \in I\right\}$ of subobjects of $M \in \mathcal{C}$ is an $a-t$ decomposition of a subobject $N$ of $M$ if and only if it is a tertiary decomposition of $N$ in $M[3, \S 5]$.

Example 8. Let $R, R^{M}$, and $\mathcal{C}$ be as in Example 1. We note that $a \leq p$ on $\mathcal{C}$ and for $M \in \mathcal{C}, N$ is an $a-p$ submodule of $M$ if and only if $N$ is a primary submodule of $M$ [4]. Furthermore, a finite set $\left\{N_{i}: i \in I\right\}$ of subobjects of $M \in \mathcal{C}$ is an $a-p$ decomposition of a subobject $N$ of $M$ if and only if it is a primary decomposition of $N$ in $M$ [4].

Example 9. Let $R$ be a commutative ring with unity and let $\mathcal{C}$ be an admissible subcategory of ${ }_{R} M$. We note that $a \leq w$ on $\mathcal{C}$ and for $M \in \mathcal{C}_{2} N$ is an $a-w$ submodule of $M$ if and only if $N$ is a weakly primary submodule of $M[3, \S 10]$. Again, a finite set $\left\{N_{i}: i \in I\right\}$ of subobjects of $M \in \mathcal{C}$ is an $a-w$ decomposition of a subobject $N$ of $M$ if and only if it is a weakly primary decomposition of $N$ in $M[3, \S 10]$.

Now we come to the main theorem in this paper.

Theorem 2.1. Let $\mathcal{C}$ be an admissible subcategory of $\mathcal{B}$ and let $r_{1}$ and $r_{2}$ be radical functions on $C$ with values in $X$ sucb that $r_{1} \leq r_{2}$. Suppose that $A$ is the associated element function on $C$ that is obtained from $r_{2}$ and suppose that for each $r_{1}-r_{2}$ subobject $N$ of $M \in \mathcal{C}, M / N$ is $r_{2}$-stable. Then necessary and sufficient conditions for $C$ to bave the $r_{1}-r_{2}$ decomposition theory are (i) each object in $C$ is $r_{2}$-worthy, and (ii) each A-stable object in $C$ is $r_{2}$-stable.

Proof. In order to prove sufficiency assume that $\mathcal{C}$ satisfies (i) and (ii). If $M \in \mathcal{C}$, then it follows from Theorem 1.3 that 0 has an $A$-decomposition $\left\{N_{i}: i \in I\right\}$ in $M$. Since each $M / N_{i}$ is $A$-stable, each $M / N_{i}$ is $r_{2}$-stable by (ii). Each $N_{i}$ is an $r_{1}-r_{2}$ subobject of $M$ since $M$ "a subobject of $M / N_{i}$ yields $r_{1}\left(M^{\prime \prime}\right) \leq$ $r_{2}\left(M^{\prime \prime}\right)=r_{2}\left(M / N_{i}\right)$. Moreover, $r_{2}\left(M / N_{i}\right) \neq r_{2}\left(M / N_{j}\right)$ for $i \neq j$ because $A\left(M / N_{i}\right) \neq$ $A\left(M / N_{j}\right)$ for $i \neq j$. Wherefore $\left\{N_{i}: i \in I\right\}$ is an $r_{1}-r_{2}$ decomposition of 0 in $M$ and therefore $\mathcal{C}$ has the $r_{1}-r_{2}$ decomposition theory.

In order to prove the necessity, assume that $C$ has the $r_{1}-r_{2}$ decomposition theory. If $\left\{N_{i}: i \in I\right\}$ is an $r_{1}-r_{2}$ decomposition of 0 in $M$, then we claim that $\left\{N_{i}: i \in I\right\}$ is an $A$-decomposition of 0 in $M$. Since each $N_{i}$ is an $r_{1}-r_{2}$ subobject of $M$, we have by hypothesis that each $M / N_{i}$ is $r_{2}$-stable. Hence each $N_{i}$ is an $A$-subobject of $M$ because $A\left(M / N_{i}\right)=\left\{r_{2}\left(M / N_{i}\right)\right\}$. Moreover $A\left(M / N_{i}\right) \neq$ $A\left(M / N_{j}\right)$ for $i \neq j$, since $r_{2}\left(M / N_{i}\right) \neq r_{2}\left(M / N_{j}\right)$ for $i \neq j$. Thus $\left\{N_{i}: i \in I\right\}$ is an $A$ decomposition of 0 in $M$ and so $\mathcal{C}$ has the $A$-decomposition theory. Consequently, each object in $\mathcal{C}$ is $r_{2}$-worthy by Theorem 1.3 .

Now suppose that $M$ is an $A$-stable object in $\mathcal{C}$. Then there exists an $r_{1}-r_{2}$ decomposition $\left\{N_{i}: i=1,2, \cdots, k\right\}$ of 0 in $M$. As above $\left\{N_{i}: i=1,2\right.$, 
$\cdots, k\}$ is an $A$-decomposition of 0 in $M$. Since $M$ is $A$-stable and $A(M)=$ $\bigcup_{i=1}^{k} A\left(M / N_{i}\right)$ [3, Proposition 4.5], we have that $k=1$. Therefore $N_{1}=0$ and so $M$ is $r_{2}$-stable. This completes the proof.

Remark. Suppose that we are in the situation of Theorem 2.1, i.e., we have a pair of radical functions $r_{1}$ and $r_{2}$ on $C$ with $r_{1} \leq r_{2} ; A$ is the associated element function on $C$ that is obtained from $r_{2}$; and for each $r_{1}-r_{2}$ subobject $N$ of $M \in \mathcal{C}, M / N$ is $r_{2}$-stable. If each object in $\mathcal{C}$ is $r_{2}$-worthy, then Theorem 1.3 guarantees that the $A$-decomposition theory exists on $\mathcal{C}$. Theorem 2.1 affords that the $r_{1}-r_{2}$ decomposition theory also exists on $\mathcal{C}$ and it coincides with the $A$-decomposition theory on $\mathcal{C}$ when and only when each $A$-stable object in $\mathcal{C}$ is $r_{2}$-stable.

Now we apply Theorem 2.1 to the tertiary, primary, and weakly primary decomposition theories for certain categories of modules. For these categories the complicating hypotheses in Theorem 2.1 melt away and leave uncomplicated existence theorems. We begin with the existence theorem for the tertiary decomposition theory which is found in Fisher [3, Theorem 5.4].

Corollary 2.2. Let $R$ be an arbitrary ring and let $\mathcal{C}$ be an admissible subcategory of $R^{M}$. Then a necessary and sufficient condition that the tertiary decomposition theory exists on $\mathcal{C}$ is that each object in $\mathcal{C}$ be t-wortby.

Proof. From Examples 2 and 7 we have a pair of radical functions, $a$ and $t$ on $\mathcal{C}$ with $a \leq t, T$ is the associated element function on $\mathcal{C}$ that is obtained from $t$, and for each $a-t$ subobject $N$ of $M \in \mathcal{C}, M / N$ is $t$-stable [3, Theorem 5.3]. Moreover, each $T$-stable object in $\mathcal{C}$ is $t$-stable [3, Theorem 5.3]. Therefore a necessary and sufficient condition for $\mathcal{C}$ to have the tertiary $(a-t)$ decomposition theory is that each object in $\mathcal{C}$ be $t$-worthy.

As another corollary of Theorem 2.1, we have the following existence theorem for the primary decomposition theory which is found in Fisher [4, Theorem 1.7].

Corollary 2.3. Let $R$ be either a commutative ring or a left Noetherian ring and let $\mathcal{C}$ be an admissible subcategory of ${ }_{R} \pi$. Then necessary and sufficient conditions for $\mathcal{C}$ to bave the primary decomposition theory are (i) eacb object in $\mathcal{C}$ is p-wortby, and (ii) each P-stable object in $C$ is p-stable.

Proof. From Examples 3 and 8 we have a pair of radical functions, $a$ and $p$ on $\mathcal{C}$ with $a \leq p, P$ is the associated element function on $\mathcal{C}$ that is obtained from $p$, and for each $a-p$ subobject $N$ of $M \in \mathcal{C}, M / N$ is $p$-stable [4, Proposition 1.5]. Therefore necessary and sufficient conditions for $\mathcal{C}$ to have the primary $(a-p)$ decomposition theory are (i) and (ii).

As another corollary of Theorem 2.1, we have the following existence theorem for the weakly primary decomposition theory. First a lemma. 
Lemma 2.4. Let $R$ be a commutative ring with unity and let $M \epsilon_{R} \mathbb{M}$. Then 0 is a weakly primary submodule of $M$ if and only if $M$ is w-stable.

Proof. Suppose that 0 is a weakly primary submodule of $M$ and $N$ is a submodule of $M$. Evidently $w(M) \subseteq w(N)$. If $x \in w(N)$, then there exists a nonzero submodule $M^{\prime \prime}$ of $M$ such that $x \in a(M ") \subseteq w(M)$.

For the "if" suppose that $M$ is $w$-stable. If $M$ " is a nonzero submodule of $M$ and $y \in a\left(M^{\prime \prime}\right)$, then $y \in a\left(M^{\prime \prime}\right) \subseteq w\left(M^{\prime \prime}\right)=w(M)$. Consequently 0 is a weakly primary submodule of $M$.

Corollary 2.5. Let $R$ be a commutative ring with unity and let $\mathcal{C}$ be an admissible subcategory of $R^{M}$. Then necessary and sufficient conditions for $\mathcal{C}$ to bave the weakly primary decomposition theory are (i) each object in $\mathcal{C}$ is w-worthy, and (ii) each W-stable object in $\mathcal{C}$ is w-stable.

Proof. From Examples 4 and 9 we have a pair of radical functions, $a$ and $w$ on $\mathcal{C}$ with $a \leq w, W$ is the associated element function on $\mathcal{C}$ that is obtained from $w$, and for each $a-w$ subobject $N$ of $M \in \mathcal{C}, M / N$ is $w$-stable by Lemma 2.4. Therefore, necessary and sufficient conditions for $\mathcal{C}$ to have the weakly primary $(a-w)$ decomposition theory are (i) and (ii).

Corollary 2.6. Let $R$ be a commutative Noetherian ring with unity and let $\mathcal{C}$

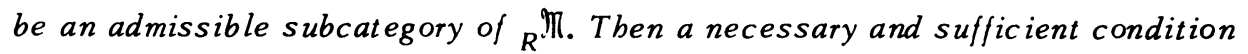
for $\mathcal{C}$ to bave the weakly primary decomposition theory is that each object in $\mathcal{C}$ is w-worthy.

Proof. Since $R$ is Noetherian, we have from Proposition 10.3 in [3] that each $W$-stable object in $\mathcal{C}$ is $w$-stable. Consequently the result follows from Corollary 2.5.

Remark. Proposition 1 in $[1$, p. 139] shows that for $R$ commutative Noetherian with unity, Bourbaki's concept of $P$-primary coincides with weakly primary. Therefore Corollary 2.6 is an existence theorem for Bourbaki's $P$-primary decomposition theory.

Now we head toward one final corollary of Theorem 2.1 .

Lemma 2.7. Let $R$ be a commutative Noetherian ring with unity and let $M \epsilon$ $R^{M}$. Then eacb nonzero submodule $N$ of $M$ contains a w-stable submodule.

Proof. The set $\{(0: x): 0 \neq x \in N\}$ has a maximal element $(0: n)$ and $(0: n)=$ $(0: R n)$. Then $R n$ is $w$-stable since the maximality of $(0: n)$ forces $(0: n)=$ $(0: r n)$ for each $0 \neq r n \in R n$.

If $R$ is a commutative ring with unity, and $M \in \epsilon_{R}^{M}$, then following Bourbaki $[1$, p. 131] we define Ass $(M)=\{\mathscr{P}: \mathcal{P}$ is a prime ideal in $R$ for which there exists $0 \neq m \in M$ such that $\underline{\mathcal{P}}=(0: m)\}$. 
Lemma 2.8. Let $R$ be a commutative Noetherian ring $w$ ith unity and let $M \epsilon$ $R^{M}$. Then $W(M) \subseteq$ Ass $(M)$.

Proof. Let $\mathcal{P} \in W(M)$. Then there exists a $w$-stable submodule $S$ of $M$ such that $\mathcal{P}=w(S)$. Again the set $\{(0: x): 0 \neq x \in S\}$ has a maximal element $(0: s)$ and $(0: s)=(0: R s)$. Since $S$ is $w$-stable, $\mathcal{P}=w(R s)$. We assert that $\mathcal{P}=(0: s)$. Obviously $(0: s) \subseteq \mathcal{P}$. If $y \in \mathscr{P}=w(R s)$, then there exists a positive integer $n$ such that $y^{n} \in(0: s)$. However, $(0: s)$ is prime. Hence $y \in(0: s)$.

Corollary 2.9. Let $R$ be a commutative Noetherian ring with unity and let $\mathcal{C}$ be an admissible subcategory of ${ }_{R}$ M. A necessary and sufficient condition for $C$ to bave Bourbaki's $\mathcal{P}$-primary decomposition theory is that Ass $(M)$ is finite for each $M \in \mathcal{C}$.

Proof. Proposition 1 in $[1$, p. 139] shows that for $R$ commutative Noetherian with unity, the concepts of $\mathcal{P}$-primary and weakly primary are equivalent. Therefore, in order to prove the sufficiency, Corollary 2.6 shows that it is enough to prove that each $M \in \mathcal{C}$ is $w$-worthy. Lemma 2.7 shows that each quotient object $M$ " of $M$ satisfies the property that each nonzero subobject of $M$ " contains a $w$ stable subobject. That $W\left(M^{\prime \prime}\right)$ is finite follows from Lemma 2.8 and the hypothesis.

The necessity follows from [1, Proposition 4, p. 144].

\section{REFERENCES}

1. N. Bourbaki, Éléments de mathématique, Fasc. XXVII. Algèbre commutative, Actualités Sci. Indust., no. 1293, Hermann, Paris, 1961. MR 30 \#2027.

2. I. Bucur and A. Deleanu, Introduction to the theory of categories and functors, Pure and Appl. Math., vol. 19, Interscience, New York, 1968. MR 38 \#4534.

3. J. W. Fisher, Decomposition theories for modules, Trans Amer. Math. Soc. 145 (1969), 241-269. MR 40 \#5656.

4. - The primary decomposition theory for modules, Pacific J. Math. 35 (1970), 359-367. MR $43 \# 264$.

5. J. W. Gray, Sheaves with values in a category, Topology 3 (1965), 1-18.

6. A. Grothendieck, Sur quelques points d'algèbre homologique, Tôhoku Math. J. (2) 9 (1957), 119-221. MR 21 \#1328.

DEPARTMENT OF MATHEMATICS, UNIVERSITY OF TEXAS, AUSTIN, TEXAS 78712 